\title{
A SEARCH FOR HI IN STRONG RADIO GALAXIES
}

\author{
S. M. SIMKIN AND J. CALLCUT \\ Michigan State University \\ East Lansing, MI 48824-1116, USA
}

\section{Introduction}

In the past decade it has become clear that many classical, double radio galaxies have hosts with extensive ionized gas inside the body of the optical galaxy (Hansen et al., 1987) (Baum et al., 1988). We have observed three such sources (PKS349-27, PKS634-20, and Pictor A) in both $\mathrm{H} \alpha$ and HI (with the VLA).

\section{H $\alpha$ and VLA measurements}

The $\mathrm{H} \alpha$ images were obtained in $1987 / 88$ with the CTIO $1.5 \mathrm{M}$ telescope using narrow band filters for both $\mathrm{H} \alpha$ and the adjacent continuum measurements. The continuum was subtracted from the line filter images and the $\mathrm{H} \alpha$ calibrated in two ways: (a) By comparing the image's nuclear flux with a combined $\mathrm{H} \alpha+[\mathrm{NII}]$ emission line flux obtained from spectra which we took with the CTIO $4 \mathrm{~m}$ during the same observing run (b) By using measurements of flux-standards taken through the same filter-ccd combination. Both methods have multiple sources of possible error (which are difficult to estimate) but agree to within a factor 2 .

The VLA observations were taken in 1989 and 1991 with the array in the $\mathrm{C} / \mathrm{D}$ configuration. The observing band width was set to cover the optical rotation velocity width (see Table 1 ). The continuum was subtracted from the uv data base and the resulting residual fluxes plotted as spectra. In no case did we detect $\mathrm{HI}$ in either emission or absorption.

\section{Analysis and Conclusions}

Table 1 lists the observed $\mathrm{H} \alpha$ flux and the calculated HII mass derived from these measurements (columns 2 and 3). We have assumed distances 
of 198, 166, and $106 \mathrm{Mpc}$ for the three objects (PKS 349-27, PKS 634-20, and Pictor A). The range in calculated masses depends on the assumed filling factors for the ionized gas. However, in all reasonable cases (filling factors of 1 to $10 \%$ ) the ionized HII exceeds $10^{9} \mathrm{M}_{\odot}$.

TABLE 1. HI and HII Parameters

\begin{tabular}{lccccc}
\hline OBJECT & $\begin{array}{c}\mathrm{F}_{\alpha}\left(10^{40}\right) \\
\left(\mathrm{erg} \mathrm{s}^{-1}\right)\end{array}$ & $\begin{array}{c}\mathrm{M}_{\alpha} / \mathrm{M}_{\odot} \\
\left(10^{9}\right)\end{array}$ & $\begin{array}{c}\Delta \mathrm{V} \\
\left(\mathrm{Km} \mathrm{s}^{-1}\right)\end{array}$ & $\begin{array}{c}\mathrm{M}_{H I} / \mathrm{M}_{\odot} \\
\left(10^{9}\right)\end{array}$ & $\mathrm{M}_{\alpha} / \mathrm{M}_{H I}$ \\
\hline P349 (nuc) & $3-6$ & $3-6$ & 47 & 1 & $3-6$ \\
P349 (50x110") & $15-30$ & $20-40$ & 800 & $0.4-0.8$ & $25-100$ \\
P634 (nuc) & $1.3-2.6$ & $0.4-0.8$ & 46 & $0.3-0.5$ & $1-3$ \\
P634 (70x70") & $13-26$ & $15-30$ & 400 & $0.2-0.4$ & $40-150$ \\
PicA (nuc) & $6-10$ & $0.1-0.2$ & 11 & $0.06-0.2$ & $0.5-3$ \\
PicA (10x10") & $13-26$ & $1-2$ & 190 & $0.3-1$ & $1-7$ \\
\hline
\end{tabular}

The VLA beam size was large relative to the optical extent of the $\mathrm{H} \alpha$ in Pictor A (see column 1); $110 \mathrm{~N}-\mathrm{S}$ x $50 \mathrm{E}-\mathrm{W}$ " for Pictor A but less for PKS 349-27 (36 x 26"), and PKS 634-20 (33 x 24"). We integrated our VLA measurements over the outer regions of these latter two galaxies to try and detect any extended HI. These are listed in the second row for each object. We have estimated three-sigma limits for our VLA HI observations from the rms noise in the residual maps. These estimates, along with the applicable velocity widths (listed in column 4), were used to calculate one of the upper limits to the HI for these objects. Since these noise limits are almost certainly an over-estimate, we also used spectra derived from the continuum-subtracted data to estimate a more stringent upper limit. The limits to the HI mass for each object (both in the nuclear regions and integrated over the disk) are given in column 5 . The resulting minimum ratios of HII to HI mass are listed in column 6.

In all three objects there is far less HI than HII. We need much higher resolution $21 \mathrm{~cm}$ observations to set good upper limits for the $\mathrm{HI}$ in the nuclear regions of these objects, but these present observations are sufficient to establish that the extended gas outside the nucleus is almost fully ionized.

\section{References}

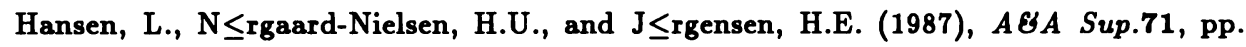
465-491

Baum, S.A., Heckman, T., Bridle, A., van Breughl, W. and Miley, G. (1988), Ap. J. Sup. 68, pp. 643-714 